\title{
Dohrenbusch/Merten/Kutzner: \\ Psychologische Begutachtung in der \\ Berufsunfähigkeitsversicherung
}

\author{
Referenzverlag Frankfurt, 2014, ISBN 978-3-943441-14- \\ $7,640 \mathrm{~S}$.
}

\section{Stephan Becher}

Online publiziert: 28. August 2014

(C) Springer-Verlag Berlin Heidelberg 2014

Die Bewertung psychischer Erkrankungen im Hinblick auf eine Berufsunfähigkeit stellt für die Private Versicherungswirtschaft die größte Herausforderung bei der Begutachtung dar. Im Rahmen dieser Fragestellung kommt der psychologischen Begutachtung unter Verwendung psychometrischer Testverfahren eine hohe Bedeutung zu. Im vorliegenden Werk werden in 6 Kapiteln nebst einem Anhang der Kontext der Begutachtung, die psychologische und neuropsychologische Begutachtung, die neuen Leitlinien und psychologische Begriffe und last but not least die häufigsten psychologischen Testverfahren vorgestellt. Besonders die prägnante Darstellung dieser Testverfahren gibt dem Leser einen sehr guten Überblick über Aussagekraft und Grenzen der Verfahren und wann solche Verfahren indiziert sind bzw. sinnbringend im gutachterlichen Kontext eingesetzt werden können. Den erfahrenen Autoren ist es zu verdanken, dass sie im vorliegenden Buch auf die Essentials eines psychologischen Gutachtens hinweisen, wie die Bestimmung des prämorbiden Leistungsniveaus, die Mitteilung von Roh- und Normwerten der Testverfahren, die Aufdeckung negativer Antwortverzerrung und deren Auftretenshäufigkeit. In diesem Zusammenhang ist die Darstellung der Beschwerdevalidierungstests, die Konsistenz - und Plausibilitätsprüfung wesentlicher Bestandteil eines solchen Gutachtens und deren ausführliche Darstellung im Werk.

Mit gut gegliederten Untertiteln wird die Thematik umfassend dargestellt. Für den Leistungsprüfer in der Versicherungswirtschaft wird damit eine Lücke geschlossen, die neben der psychiatrischen Begutachtung bisher in dieser Form und diesem Umfang nicht vorlag. Es kann allen Mitarbeitern in den Versicherungsgesellschaften, die sich mit Fragen der Leistungsregulierung beschäftigen uneingeschränkt empfohlen werden.

S. Becher $(\bowtie)$

Köln, Deutschland

E-Mail: sbecher@scor.com 